\title{
USING A DRIVING SIMULATOR TO CREATE A VISUAL SEARCH TEST FOR DRIVERS WITH PARKINSON'S DISEASE
}

\author{
Hannes Devos ${ }^{1}$, Maud Ranchet ${ }^{2}$, John C. Morgan ${ }^{3}$, Abiodun E. Akinwuntan ${ }^{1}$ \\ ${ }^{1}$ University of Kansas Medical Center, Kansas City, KS, USA \\ ${ }^{2}$ IFSTTAR, Bron, France, ${ }^{3}$ Augusta University, Augusta, GA, USA \\ Email: hdevos@kumc.edu
}

\begin{abstract}
Summary: Visual search has been reported as one of the most important determinants of on-road driving in Parkinson's disease (PD). Yet, commonly used visual search tests are administered on paper or on a computer and have no to little face validity. This study aimed to (1) create a visual search test in a driving simulator; (2) investigate the convergent validity of the test against the dot cancellation (DC) test; and (3) compare performance on the test between 20 drivers with PD and 15 controls. Participants searched for a target road sign among a clutter of other road signs on three screens with $100^{\circ}$ of horizontal visual field. Drivers with PD took longer to respond $(9 \mathrm{~s} \pm 2 \mathrm{vs} 7 \mathrm{~s} \pm 1 ; \mathrm{p}=0.001)$ and missed more target road signs $(1.50(0.5-7)$ vs $0(0-1) ; \mathrm{p}=0.01)$ than controls. No differences were found between groups on the DC test. Response time on the visual search test correlated strongly with DC time $(r=0.52 ; \mathrm{p}=0.009)$ and moderately with DC errors $(\mathrm{r}=0.37 ; \mathrm{p}=0.03)$. Missed responses correlated moderately with DC time $(\mathrm{r}=0.49 ; \mathrm{p}=0.02)$. Our findings suggest that the driving simulator visual search test offers a valid alternative to standard visual search tests. Future research is needed to investigate the validity of the new visual search test in predicting on-road driving performance in PD.
\end{abstract}

\section{INTRODUCTION}

Visual search is one of the most important skills for safe driving. Drivers constantly search for road signs, other vehicles, potential hazards, and pedestrians to adapt their driving behavior. Visual search may be compromised in patients with Parkinson's disease (PD) (Archibald et al., 2013). Missing critical information from deficits in visual search may inhibit the ability to successfully scan the environment for potential problems, thus decreasing on-road driving performance (Classen et al., 2014; Devos et al., 2013; Uc et al., 2006). Drivers with PD identify fewer road signs and landmarks when compared with healthy controls (Uc et al., 2006). Furthermore, they make more critical on-road driving errors while engaged in a visual search task compared to controls (Uc et al., 2006).

Driving rehabilitation specialists and health professionals (psychologists, occupational therapists, physical therapists, medical doctors) often assess driving ability with a battery of off-road visual, motor, or cognitive tests, and a practical on-road test. The off-road battery typically assesses driving-related visual sensory functions (e.g., visual acuity), motor functions (e.g., strength, range of motion), and cognitive functions (e.g., memory, attention, perceptual, visuospatial and executive functions). Performance on these tests informs the examiner which deficits to focus on during the on-road driving test. Off-road visual search performance emerged as the most significant predictor of on-road driving performance in PD in our previous study (Devos et al., 2013). However, off-road visual search tests are usually administered on paper or on a computer 
and bear few similarities with actual driving. For example, the dot cancellation (DC) requires participants to cross out groups of four dots on an A4 sheet of paper (Nouri \& Lincoln, 1993). In the computerized visual scanning test from the Zimmerman and Fimm (2018) battery, participants are asked to identify a square with an opening on top amongst a 5 x 5 matrix of other squares on a single monitor. Although these tests are important predictors of driving in neurological conditions (Akinwuntan et al., 2002, Devos et al., 2013, Nouri \& Lincoln, 1993), there is a need to create visual search tests that better resemble real-world driving.

The purpose of the current study was to create a new visual search test on a driving simulator that provides a context similar to visual search during real-life driving. We explored the ability of our new test to discriminate between drivers with PD and healthy controls, and tested the convergent validity of the new visual search test with performance on the DC test. The DC test is part of the Stroke Drivers Screening Assessment and has been widely validated as an accurate predictor of fitness-to-drive in several neurological conditions (Nouri \& Lincoln, 1993; Radford et al., 2004).

\section{METHOD}

\section{Participants}

Twenty-two patients were recruited from the outpatient Movement Disorders Clinic at Augusta University and 17 controls were recruited from the community. Among them, 20 participants with PD and 15 healthy controls were included (Table 1). Two patients were excluded from the analyses due to difficulties understanding the test. Two controls did not complete the test. Inclusion criteria were: (1) diagnosis of idiopathic PD (for the PD group); (2) valid driver license; and (3) driving more than 500 miles/year. Exclusion criteria were: (1) presence of dementia by clinician impression (J.C.M.); (2) Montreal Cognitive Assessment (MOCA) scores $\leq 15$; (3) other parkinsonian syndromes; (4) severe dyskinesia; (5) deep brain stimulation; (6) unpredictable motor fluctuations; and (7) significant diseases causing visual impairment (glaucoma, stroke, eye vascular disorders, macular degeneration, etc.).

Binocular acuity (range: 0 -1) was assessed by the vision screening apparatus from Keystone view (Visionary Software version 2.0.14). L-Dopa equivalent dosages (LED) and motor scores on Unified Parkinson's Disease Rating Scale (UPDRS III) were recorded for all participants with PD. All but one patient were medicated and were tested in the "on" medication state. The study was approved by the institutional review board of Augusta University. Informed written consent was obtained from all participants.

\section{Driving Simulator Set-up}

The desktop driving simulator M100WS with three 22" screens (100 degrees of combined horizontal field of view and 20 degrees vertical field of view) was powered on STISIM drive ${ }^{\circledR}$ software, version 3 (STI Inc, Hawthorne, CA). A Logitech ${ }^{\circledR}$ steering wheel and pedals were connected to the computer. Only the buttons on the top right and top left were functional.

\section{Creation of Visual Search Test}

The standard STISIM Drive ${ }^{\circledR}$ for Logitech ${ }^{\circledR}$ steering wheel was reconfigured to remove all the roadway section parameters and remove the sound of the car engine. The background, ground 
plane, and other default textures were changed to RGB colors 0/0/0 (black). Although no car or driving scene would be viewed on the screen, we needed to set a fixed speed of an invisible car driving in the background since the events in the STISIM Drive ${ }^{\circledR}$ scenario definition language were distance-based and not time-based. Therefore, the automated pilot function of the invisible car was checked in the configuration settings and set at 66 feet per second.

The simulator-based visual search test involved the presentation of different road signs that were projected in the central and peripheral field of view (Figure 1). We used European road signs for US drivers to avoid knowledge of road signs to influence performance on the visual search test. Participants were instructed to first focus on the middle screen and notice a specific and bigger road sign presented in the center of the screen. This sign is designated the central road sign. Participants were then instructed to search among many other different smaller road signs, i.e., distractors, to identify if the road sign displayed in the center of the screen is present or absent among the distractors displayed in the periphery of the screens. The road sign displayed in the periphery that is identical to the central road sign is the target road sign. The central road sign changed for each trial. The distractor road signs were also displayed randomly across each trial.

The visual search test consisted of 42 trials. The target sign was present among the distractors during 21 of the trials as shown in Figure 1. When present, the target sign was presented randomly at 22 different locations that varied from $5^{\circ}$ to $50^{\circ}$ of horizontal visual angle from the central target. Participants were instructed to press the right button when the target sign was present and to press the left button in the absence of the target sign. In all cases, when the participant pressed either the right or left button, a black screen appeared and the next trial started. The maximum duration of each of the 42 trials was 15 seconds. The total duration of the test was 15 minutes.

The four outcome variables extracted for this experiment were average response time, total number of missed responses, number of no detections and number of false alarms. Response time was averaged only on responses within the 15 -second window. No responses or responses outside the 15 -second window were considered missed responses. No detections were defined as trials when a target was present, but the participant pressed the wrong (left) button). False alarms were defined as trials when the target was absent, but the participant pressed the wrong (right) button). Data were collected at $60 \mathrm{~Hz}$.

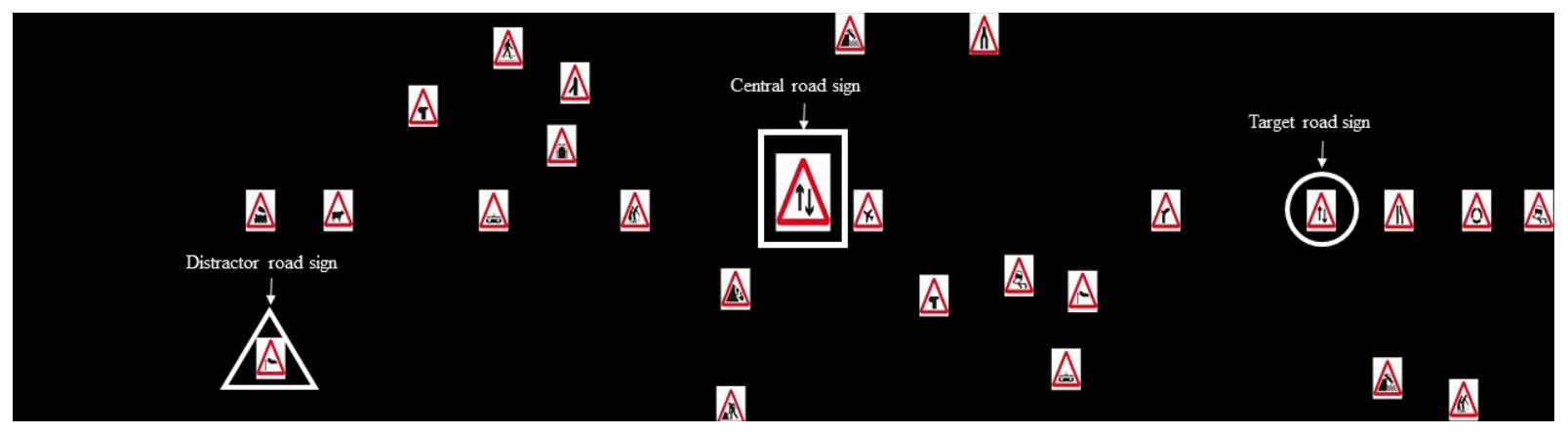

Figure 1. Visual search test in driving simulator 


\section{Dot Cancellation Test}

In the dot cancellation test, participants were handed a sheet of A4 paper with 25 rows and 25 columns of groups of three, four, or five dots (Figure 2). Participants were instructed to read each line (with the first line as an example line) and mark all groups of four dots, while ignoring the groups of three dots and five dots. The maximum allotted time to complete the test was 15 minutes. Total time, number of errors (missed responses) and false positives were recorded.

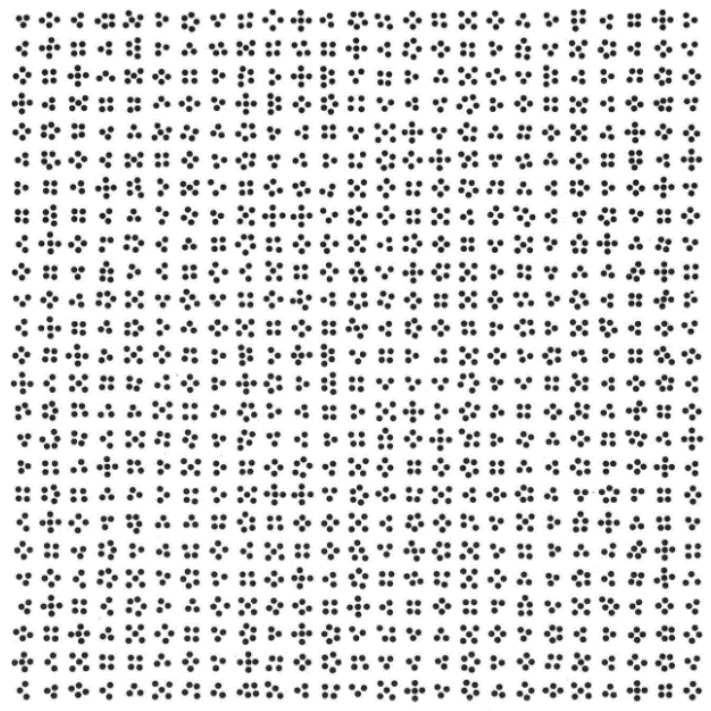

Figure 2. Dot cancellation test

\section{Data Analysis}

Between-group differences were examined using Fisher's Exact tests, student t tests or Wilcoxon rank-sum tests as appropriate based on the Kolmogorov-Smirnov normality testing. Cohen's d effect sizes were calculated for the driving simulator outcome variables and the dot cancellation test. Effect sizes (ES) between 0.50 and 0.80 were considered medium, whereas ES larger than 0.80 were considered large. Since age differed significantly between groups, univariate analyses of covariance (ANCOVA) or generalized linear models with age as covariate and group as between-group factor were performed to investigate the effect of group status (PD vs controls) on performance in the visual search tests. Partial Pearson correlations (r) were analyzed to investigate associations between response time and missed responses of the driving simulator visual search test with time, errors, and false positives on the dot cancellation test, whilst controlling for age. Correlation coefficients between 0.10 and 0.29 were considered as weak, those between 0.30 and 0.49 were moderate, and those $\geq 0.50$ were strong in terms of magnitude (Cohen, 1992). P values $<0.05$ were considered significant. All statistical analyses were performed with SPSS, version 25.0.

\section{RESULTS}

\section{Demographic and Clinical Characteristics}

Patients were significantly older than controls and performed worse on the MOCA, even after adjusting for age $(p=0.003)$. Since age was significantly correlated with $\operatorname{MOCA}(r=-0.38 ; p=$ 
0.02), we only controlled for differences in age in the subsequent analyses. Both groups had normal-to-corrected vision. The Hoehn and Yahr stages and the scores on the UPDRS III showed that patients were in the mild to moderate stage of the disease.

Table 1. Demographic and clinical characteristics

\begin{tabular}{|c|c|c|c|}
\hline Variable & $\begin{array}{l}\text { PD group } \\
(\mathrm{n}=20)\end{array}$ & $\begin{array}{c}\text { Control group } \\
(\mathrm{n}=15)\end{array}$ & Test, $\mathrm{p}$ value \\
\hline Age, years & $69 \pm 8$ & $61 \pm 11$ & $\mathrm{t}, \mathbf{0 . 0 3}$ \\
\hline Sex (male), n \% & $16(80)$ & $8(47)$ & Fisher, 0.07 \\
\hline Education, years & $17(16-18)$ & $15(13-20)$ & W, 0.49 \\
\hline $\begin{array}{l}\text { MOCA score, } \\
\quad(0-30)\end{array}$ & $26(20-27.5)$ & $29(27-30)$ & $\mathrm{W}, \mathbf{0 . 0 0 1}^{\mathrm{a}}$ \\
\hline Visual acuity, (0-1) & $0.8(0.5-0.8)$ & $0.8(0.7-1)$ & $\mathrm{W}, 0.27$ \\
\hline Disease duration, years & $5(2-7)$ & NA & \\
\hline LED, mg/day & $340(250-733)$ & NA & \\
\hline $\begin{array}{l}\text { Hoehn and Yahr stage } \\
\text { (on) }\end{array}$ & $2(2-3)$ & NA & \\
\hline UPDRS motor (on) & $31(19.5-38.5)$ & NA & \\
\hline
\end{tabular}

\section{Visual Search Performance}

\section{Driving Simulator}

Significant differences in response times and in missed responses were found between patients and controls (Table 2). Participants with PD took longer to respond $(E S=1.26 ; p=0.001)$ and missed more target signs ( $E S=0.73 ; p=0.01)$ compared to the control participants. No differences between groups were observed for errors (i.e., no detections and false alarms).

\section{Dot Cancellation}

No significant differences between groups were found on any of the three DC variables (Table 2). DC time reached borderline significance $(E S=0.91 ; p=0.08)$.

\section{Correlations between Visual Search in Driving Simulator and Dot Cancellation}

Partial correlations between visual search outcome variables in the simulator and on the DC test were adjusted for age. Response time on the visual search test correlated strongly with DC time $(\mathrm{r}=0.52 ; \mathrm{p}=0.009)$ and moderately with $\mathrm{DC}$ errors $(\mathrm{r}=0.37 ; \mathrm{p}=0.03)$. Missed responses on the visual search test correlated moderately with $\mathrm{DC}$ time $(\mathrm{r}=0.49 ; \mathrm{p}=0.02)$. False positives correlated strongly with DC time $(r=0.53 ; p=0.01)$. No detections showed no correlation with any of the DC outcomes.

\section{Correlations between visual search in driving simulator and clinical characteristics}

Correlations were explored between visual search outcomes and clinical characteristics in the PD group. Scores on the MOCA correlated strongly with visual search response time $(\mathrm{r}=-0.57 ; \mathrm{p}=$ $0.009)$, number of omissions $(r=-0.77 ; \mathrm{p}<0.0001)$, and number of false alarms $(\mathrm{r}=-0.56 ; \mathrm{p}=$ 
0.01). Disease duration correlated strongly with number of no detections on the visual search test $(\mathrm{r}=0.51 ; \mathrm{p}=0.03)$. Other measures of disease severity (Hoehn and Yahr stages, UPDRS, and LED) did not show strong relationships with any outcome of the visual search test.

Table 2. Differences in visual search performance between patients and controls

\begin{tabular}{ccccc}
\hline Variable & $\begin{array}{c}\text { PD group } \\
(\mathrm{n}=20)\end{array}$ & $\begin{array}{c}\text { Control group } \\
(\mathrm{n}=15)\end{array}$ & ES & $\begin{array}{c}\text { Test, } \mathrm{p} \text { value } \\
\text { adjusted for age }\end{array}$ \\
\hline Driving simulator & & & & \\
Response time, $\mathrm{s}$ & $9 \pm 2$ & $0 \pm 1$ & 1.26 & $\mathrm{~F}, \mathbf{0 . 0 0 1}$ \\
Missed responses, $\mathrm{n}$ & $1.50(0.50-7)$ & $0(0-1)$ & 0.73 & $\mathrm{X}^{2}, \mathbf{0 . 0 1}$ \\
No detections ${ }^{\mathrm{a}}, \mathrm{n}$ & $3(2-5)$ & $3(1-6)$ & 0.0006 & $\mathrm{X}^{2}, 0.99$ \\
False alarms ${ }^{\mathrm{b}}, \mathrm{n}$ & $0(0-2)$ & $0(0-0)$ & 0.43 & $\mathrm{X}^{2}, 0.50$ \\
Dot cancellation & & & & \\
Time, $\mathrm{s}$ & $587(451-883)$ & $405(356-560)$ & 0.91 & $\mathrm{X}^{2}, 0.08$ \\
Errors, $\mathrm{n}$ & $7(2-14)$ & $4(3-9)$ & 0.33 & $\mathrm{X}^{2}, 0.68$ \\
False positives, $\mathrm{n}$ & $0(0-0)$ & $0(0-0)$ & 0.18 & $\mathrm{X}^{2}, 0.66$ \\
\hline
\end{tabular}

$\mathrm{ES}=$ Cohen's d effect size; F, ANOVA; $\mathrm{X}^{2}, \mathrm{Chi}^{2}$ of generalized linear model

${ }^{a}$ number of trials with a target present where the participant pressed the wrong (left) button);

${ }^{b}$ number of trials with a target absent where the participant pressed the wrong (right) button.

\section{DISCUSSION}

The aim of this study was to create a visual search test with a wide field-of-view setting to more closely resemble visual search during driving in real traffic situations. After successfully using driving simulator software to create the visual search tests and administer the test in a driving context, the test distinguished between drivers with PD and control drivers in response time and in missed responses. Convergent validity was established by the moderate to strong correlations found between performance in the simulator visual search test and DC test. Visual search showed to correlate with scores of a general cognitive test and disease duration.

The results of the driving simulator-based visual search test showed that patients were slower to respond than controls. Slower response times may have implications for driving that requires individuals to timely respond to unanticipated hazards on the road. They also missed more targets than controls. Mannan et al. (2008) also demonstrated that patients missed more targets than controls, even though the patients in that study did not take longer to complete the test. Our findings are also consistent with the study from Uc et al. (2006) that showed that drivers with PD identified fewer landmarks and traffic signs. Interestingly, patients did not exhibit decreased performance on the DC test that was administered in a context that was not suggestive of driving. The driving simulator visual search test discriminated better between groups than the DC test. Extending the field of view to 100 degrees may evoke more difficulties with visual scanning in PD than visual scanning on a sheet of paper. In order to scan the three screens, drivers have to make larger and more eye movements (saccades), and rotate the neck and trunk. Individuals with PD typically have difficulties with saccadic movements, and show muscle stiffness (rigidity) and slow movements (bradykinesia) in the neck and trunk, which may limit their ability to scan the periphery (Archibald et al., 2013). We cannot rule out that bradykinesia and tremor in the thumb affected response times on the visual search task. However, we expect the influence of thumb bradykinesia and tremor on response time to be minimal, since the thumbs of both hands rested 
on the button. To fully discern motor versus cognitive contributions to visual search, an oral version of the visual search test should be considered.

In summary, the current findings show (1) that driving simulator software can be used to create a variety of cognitive tasks that involve visual scanning; (2) that the newly developed visual search test discriminated better between drivers with PD and controls compared with a paper-and-pencil visual scanning test; and (3) the visual search test showed convergent validity with a paper-andpencil visual scanning test. We made a conscious decision to create a visual search test, albeit in a driving simulator, without any driving background. The purpose of the newly developed test is to better understand the role of visual search impairments on on-road driving in PD. For this, we created a cognitive test that evaluated only visual search skills, ruling out any decrements in visual search performance due to impairments in executive functions, speed of processing, or psychomotor speed. However, we fully appreciate that the constantly changing and cluttered background of a driving scene will make visual search even more complicated than the test we created. In a future study, we will create a simulator-based visual search test against a moving background to even better resemble visual search skills typically employed in real-world driving.

\section{ACKNOWLEDGMENTS}

We thank the American Parkinson's Disease Association for funding this research. We also thank Adam Bruetsch, MS, for his help in data analysis.

\section{REFERENCES}

Akinwuntan, A.E., Feys, H., DeWeerdt, W., Pauwels, J., Baten, G., Strypstein, E. (2002). Determinants of driving after stroke. Archives of Physical Medicine and Rehabilitation, 83, 334-341.

Archibald, N. K., Hutton, S. B., Clarke, M. P., Mosimann, U. P., \& Burn, D. J. (2013). Visual exploration in Parkinson's disease and Parkinson's disease dementia. Brain, 136(Pt 3), 739750.

Classen, S., Brumback, B., Monahan, M., Malaty, I. I., Rodriguez, R. L., Okun, M. S., \& McFarland, N. R. (2014). Driving errors in Parkinson's disease: moving closer to predicting on-road outcomes. The American Journal of Occupational Therapy, 68(1), 77-85.

Devos, H., Vandenberghe, W., Tant, M., Akinwuntan, A. E., De Weerdt, W., Nieuwboer, A., \& Uc, E. Y. (2013). Driving and off-road impairments underlying failure on road testing in Parkinson's disease. Movement Disorders, 28(14), 1949-1956.

Nouri, F., \& Lincoln, N.B. Predicting driving performance after stroke. (1993). British Medical Journal, 307, 482-483.

Radford, K., Lincoln, N., Lennox, G. (2004). The effect of cognitive abilities on driving in people with Parkinson's disease. Disability and Rehabilitation, 26, 65-70.

Uc, E. Y., Rizzo, M., Anderson, S. W., Sparks, J., Rodnitzky, R. L., \& Dawson, J. D. (2006). Impaired visual search in drivers with Parkinson's disease. Annals of Neurology, 60(4),407413.

Zimmermann, P., Fimm, B. (2018). Test of Attentional Performance (TAP), version 2.3.1. Psychological Test Systems, Herzogenrath, Germany. 